\title{
Maximal Mean Field Solutions in the Random Field Ising Model: the Pattern of the Symmetry Breaking
}

\author{
Marco GUAGNELLI ${ }^{(a)}$, Enzo MARINARI ${ }^{(a, b)}$ \\ and \\ Giorgio PARISI ${ }^{(c)}$ \\ (a): Dipartimento di Fisica and Infn, \\ Università di Roma Tor Vergata, \\ Viale della Ricerca Scientifica, 00173 Roma, Italy \\ (b): Department of Physics and NPAC, \\ Syracuse University \\ Syracuse, NY 13244, USA \\ (a): Dipartimento di Fisica and Infn, \\ Università di Roma La Sapienza, \\ P. Aldo Moro 2, 00185 Roma, Italy \\ guagnelli@roma2.infn.it marinari@roma1.infn.it parisi@roma1.infn.it
}

March 20, 1993 


\begin{abstract}
In this note we study the mean field equations for the $3 d$ Random Field Ising Model. We discuss the phase diagram of the model, and we address the problem of finding if such equations admit more than one solution. We find two different critical values of $\beta$ : one where the magnetization takes a non-zero expectation value, and one where we start to have more than one solution to the mean field equation. We find that, inside a given solution, there are no divergent correlation lengths.
\end{abstract}

ROM2F-92-46

SCCS 372

cond-mat/9303042 


\section{Introduction}

The Random Field Ising Model (RFIM) (see for example refs. [1, 2, 3]) is waiting for pieces of new understanding and further clarifications of the relevant physical mechanisms.

Let us start by sketching the theoretical situation. For a certain time it was hoped that dimensional reduction could be the appropriate method to compute the critical behavior of a ferromagnet in presence of a random magnetic field. It was proven in [4] that in perturbation theory the sum of the most divergent diagrams close to the phase transition for a random field model in dimension $D$ coincides with that of a ferromagnetic theory, without random field, in the reduced dimension $d=D-2$. The terms that are neglected are less singular than the leading ones by a factor $\xi^{-2}, \xi$ being as usual the correlation length. This result suggests that all the exponents of the random field system coincide with those of the corresponding ferromagnetic system in $D-2$ dimensions.

Clearly this result cannot be correct. Simple physical arguments (confirmed by a rigorous analysis [5]) lead to the conclusion that the lower critical dimension is 2 , not 3 , as implied by dimensional analysis. The deep reason for this failure can be found following the non-perturbative analysis of ref. $[3,6]$. Let us summarize the main results.

We assume that the system is described by the following Hamiltonian density, which is a functional of the order parameter $\phi(x)$ :

$$
H[\phi]=\int d^{D} x\left(\frac{1}{2}(\partial \phi)^{2}+V(\phi)-h(x) \phi(x)\right),
$$

where the random field $h(x)$ is a Gaussian uncorrelated white noise with variance $g \delta(x-y)$, and $g$ parametrizes the strength of the random field.

The stationary points of $H$ can be found by solving the corresponding mean field equations

$$
-\Delta \phi+V^{\prime}(\phi)=h(x) \text {. }
$$

When these equations admit only one solution, as it happens for sufficiently large temperature, it is natural to introduce the correlations functions

$$
C(x) \equiv \overline{\phi(x) \phi(0)}
$$




$$
G(x) \equiv \overline{\frac{\delta \phi(x)}{\delta h(0)}}=\overline{\left\langle x\left|\frac{1}{\Delta+V^{\prime \prime}(\phi)}\right| 0\right\rangle},
$$

where by the long bar we denote the thermal average over all the realizations of the random magnetic fields.

These two correlation functions are the mean field approximation to $\overline{<\phi(x)><\phi(0)>}$ and $\overline{\langle\phi(x) \phi(0)\rangle_{c}}$ respectively. Then one finds that $C(x)$ is proportional to the same correlation function of the pure system in dimensions $d=D-2$. The functions $G(x)$ and $C(x)$ are related one to the other. In Fourier space one finds that

$$
\begin{aligned}
C(k) & =g \int d \mu \rho(\mu) \frac{1}{\left(k^{2}+\mu^{2}\right)^{2}} \\
G(k) & =\int d \mu \rho(\mu) \frac{1}{k^{2}+\mu^{2}} .
\end{aligned}
$$

The function $G(k)$ is the same as for the pure system in dimension $d=$ $D-2$ (dimensional reduction works in configuration space with the function $C$, and in momentum space with the function $G$ ).

When 2 admits more than one solution to compute expectation values we must assign a weight to each solution. This makes life more complicated. If we label by $\alpha$ different solutions, and by $w_{\alpha}$ the relative weight we can write

$$
\begin{aligned}
C(x) & =\sum_{\alpha} w_{\alpha} \overline{\phi(x) \phi(0)} \\
G(x) & =\sum_{\alpha} w_{\alpha} \frac{\overline{\delta \phi_{\alpha}(x)}}{\delta h(0)} \\
& =\sum_{\alpha} w_{\alpha} \overline{\left\langle x\left|\frac{1}{-\Delta+V^{\prime \prime}(\phi)}\right| y\right\rangle}
\end{aligned}
$$

By using different prescriptions for the weights $w_{\alpha}$ we can obtain different results. This is especially true if the number of different solutions of the mean field equations increases with the volume.

Dimensional reduction can still hold, but with a crazy choice of the weights: 


$$
w_{\alpha}=\frac{\operatorname{sign} \operatorname{det}\left[-\Delta+V^{\prime \prime}\left(\phi_{\alpha}\right)\right]}{Z_{w}}
$$

where $Z_{w}$ is such that $\sum_{\alpha} w_{\alpha}=1$. Here the Morse theorem states that $Z_{w}=1$.

A physically motivated choice would be:

$$
w_{\alpha}=\frac{\operatorname{sign} \operatorname{det}\left[-\Delta+V^{\prime \prime}\left(\phi_{\alpha}\right)\right] \exp \left(-\beta H\left[\phi_{\alpha}\right]\right)}{Z_{w}},
$$

where $\alpha$ runs over all the solutions of the mean field equations, minima, maxima and saddle points all together. The strange looking unusual factor sign $\operatorname{det}\left[-\Delta+V^{\prime \prime}\left(\phi_{\alpha}\right)\right]$ is needed to keep the continuity of $Z_{w}$ when new solutions appear.

It could be argued that the energy of the minima is so smaller than the energy of the saddle points and maxima, that we can simply write

$$
w_{\alpha}=\frac{\exp \left(-\beta H\left[\phi_{\alpha}\right]\right)}{Z_{w}}
$$

and keep the sum restricted only to the minima. In the rest of this paper we follow this second strategy.

It is possible that this modified mean field theory gives the correct results (as it is implicit in the work of ref. [7]) and that the failure of dimensional reduction is simply related to the existence of many solutions with different energy $([3,6,8])$.

Our aim is here to investigate numerically this improved mean field approximation to make its predictions explicit and eventually to compare them with Monte Carlo simulations. We have been motivated to start this investigation by an interesting paper [9], in which it was suggested that replica symmetry is already broken at the point ferromagnetic phase transition. For results obtained both in the mean field framework and with a Monte Carlo and a $T=0$ optimization approach, see refs. $[10,11,7]$.

In this note we limit ourselves to the study of two particular solutions of the mean fields equations, which we call $\phi_{+}$and $\phi_{-}$. They are such that for any solution $\phi_{\alpha}$ (and for any $x$ ) the relation $\phi_{-}(x) \leq \phi_{\alpha}(x) \leq \phi_{+}(x)$ holds. The existence of two solutions with this property (in the high temperature phase they coincide) follows from convexity arguments [12]. We call them maximal mean field solutions. 


\section{Lattice Mean Field Equations}

We consider the Random Field Ising Model (RFIM) with Ising type $\left(Z_{2}\right)$ variables defined on a $3 d$ simple cubic lattice. We study the solutions of its mean field equations.

With $i$ we denote the triplet of integers $(x, y, z)$, which characterize the lattice sites. We will consider configurations of the random field $\left\{h_{i} \equiv\right.$ $\left.\theta_{i} \mathcal{H}\right\}$, where the quenched random variables $\theta_{i}$ can take the values \pm 1 with probability $\frac{1}{2}$, and we have chosen the absolute value of the field, $\mathcal{H}$, to be 1.5. Such choice for $\mathcal{H}$ was meant to allow the critical temperature $T_{c}$ to have a non negligible shift from $T_{c}$ in the pure model, and simultaneously not to be large enough to allow the transition to become first order ${ }^{[1]}$.

In the mean field approximation one introduces local magnetization variables $m_{i}$, which play the same role of $\phi(x)$ in the continuum formalism. The total free energy is written as a function of the local magnetization, and the condition for the free energy being stationary is the usual mean field equation

$$
m_{i}=\tanh \left(\beta\left(\mathcal{D} m_{i}+h_{i}\right)\right),
$$

where with $\mathcal{D} m_{i}$ we define the lattice sum over the 6 first neighbor variables.

If this equation admits only one solution there is no ambiguity. If, on the contrary, there are many solutions, one has to weight (according to the previous discussion) different solutions with a weight proportional to the exponential of minus the free energy (multiplied by $\beta$ ).

Our ideal goal is to look for all solutions of this equation, which correspond to local minima of the free energy, but this is an awful task when the number of solutions is very large, as it happens at low $T$. Here we have just looked for the solutions with higher, positive and negative, magnetization, $\left(m_{+}\right.$and $m_{-}$) using a simple iterative scheme. We have started the iterative procedure used to solve eq. (9) from the two initial conditions $m_{i}=m_{s}$ and $m_{i}=-m_{s}$. Although a completely safe procedure would start from $m_{s}=1$, it is more convenient (and it does not change the results) to take a value for $m_{s}$ slightly smaller than one. The appropriate value of $m_{s}$ depends on the temperature; in our simulations we have taken $m_{s}=.6$.

In the high $T$ regime both these runs converge to the same (unique) solution. In a broken phase they will tend to different solutions with average magnetization of opposite signs. This procedure should be good enough to 
localize the temperature $T$ below which the solution of (9) is not unique, and to give relevant quantitative hints about the structure of the phase transition.

We label the solutions of the mean field equations, in a given realization of the magnetic field, by the index $\alpha$; given the pattern of our search $\alpha$ is limited to take only one or two values. For each realization of the magnetic field the index $\alpha$ belongs to the set $A$ (which can be, in our simulation, constituted of 1 or 2 solutions). The average over different field samples (which we denote by a bar: we denote the average over different solutions by $\langle\cdot\rangle)$ is done by having $A$ running from 1 to $N_{A}$.

In each solution $\alpha$ (characterized by the $V \equiv L^{3}$ values of the local magnetization $m_{i}$ ) we compute the relevant observables. We define the total magnetization density

$$
m^{\alpha} \equiv \frac{1}{V} \sum_{i} m_{i}^{\alpha}
$$

and the sum of the squared local variables

$$
q^{\alpha} \equiv \frac{1}{V} \sum_{i}\left(m_{i}^{\alpha}\right)^{2}
$$

We define the energy density

$$
E^{\alpha} \equiv-\frac{1}{V} \sum_{i}\left(\frac{1}{2} m_{i}^{\alpha} \mathcal{D} m_{i}^{\alpha}+h_{i} m_{i}^{\alpha}\right),
$$

the entropy density

$$
S^{\alpha} \equiv-\frac{1}{V} \sum_{i}\left(\frac{1+m_{i}^{\alpha}}{2} \log \left(\frac{1+m_{i}^{\alpha}}{2}\right)+\frac{1-m_{i}^{\alpha}}{2} \log \left(\frac{1-m_{i}^{\alpha}}{2}\right)\right)
$$

and the total free energy as

$$
F^{\alpha}=V\left(\beta E^{\alpha}-S^{\alpha}\right) .
$$

The weight $w_{\alpha}$ associated to each solution $\alpha$ is given by

$$
w_{\alpha}=\frac{\exp \left(-\beta F^{\alpha}\right)}{Z_{w}} .
$$




\section{$3 \quad$ Numerical Results for Local Quantities}

Here we present numerical results for system of size up to 48 in a range of $\beta$ that goes from 1.1 to 1.5 (we will always give $\beta$ in units of the critical $\beta$ at zero random field, i.e., $\frac{1}{6}$ ). We have analyzed 600 random field samples for the $12^{3}$ lattice, 400 for the $24^{3}$ lattice, 200 for the $36^{3}$ lattice and 30 for the $48^{3}$ lattice.

In this section we will discuss the behavior of local quantities (i.e., those objects that can easily be constructed from the magnetization), while in the next section we will concentrate our attention on the response functions, which must be computed by inverting the lattice equivalent of $\left(-\Delta+V^{\prime \prime}(\phi)\right)$, a highly non-local operation.

A very interesting quantity is

$$
W^{2} \equiv \sum_{\alpha} w_{\alpha}^{2}
$$

This quantity is different from 1 when the mean field equations admit more than one solution: roughly speaking $W^{-2}$ is the average number of relevant solutions. We display the results for $W^{2}$ as function of $\beta$ in fig. 1. We see that $W^{2}$ becomes sizably different from 1 only at $\beta$ greater than 1.2 . We see a change in regime at this beta, which we denote by $\beta_{1}$.

Another quantity that is interesting to measure is the maximal magnetization $m_{M}^{2}$, defined as $\max _{\alpha}\left(m^{\alpha}\right)^{2}$. In fig. 2 we show the $\beta$ dependence of $m_{M}^{2}$ for different lattice sizes. We see a transition from an asymptotic zero value of $m_{M}^{2}$ to a non zero value around $\beta=1.35$. The transition becomes sharper by increasing the size of the lattice. We see a change in regime also at this new value of $\beta$, which we denote $\beta_{2}$.

A more detailed understanding can be obtained by considering the correlation functions of the local magnetization. At this end we define, for each solution $\alpha$, the magnetization on a 2-plane as

$$
M_{x}^{\alpha}(\lambda) \equiv \sum_{y, z} m^{\alpha}(\lambda, y, z)
$$

$M_{y}^{\alpha}(\lambda)$ and $M_{z}^{\alpha}(\lambda)$ are defined in an analogous way. We define the zero (bi-)momentum magnetization-magnetization correlation function for the solution $\alpha$ as 


$$
C^{\alpha}(\lambda) \equiv \sum_{\mu=x, y, z ; \lambda_{1}, \lambda_{2}} \sum_{\text {such that }\left|\lambda_{1}-\lambda_{2}\right|=\lambda} M_{\mu}^{\alpha}\left(\lambda_{1}\right) M_{\mu}^{\alpha}\left(\lambda_{2}\right) .
$$

The total correlation function at distance $\lambda$, averaged over $N_{A}$ samples, is defined as

$$
C(\lambda) \equiv \frac{1}{N_{A}} \sum_{A} \sum_{\alpha} w^{\alpha} C^{\alpha}(\lambda),
$$

and we denote by $C_{c}(\lambda)$ its connected part.

At first order in perturbation theory in the strength of the random field $[3,6,4] C_{c}(\lambda)$ has (as we have discussed before) a double pole in Fourier space. It has also been shown that this form retains its validity at all orders in perturbation theory, and should be exact in the region where supersymmetric considerations hold. In $x$ space that leads to

$$
C_{c}(\lambda) \simeq A\left(1+\frac{\lambda}{\xi^{(C)}}\right) e^{-\frac{\lambda}{\xi^{(C)}}}+B,
$$

that defines the correlation length $\xi^{(C)}$.

In fig. 3 we plot the inverse correlation length as a function of $\beta$. We have used a global fit to $C(\lambda)$ (which has determined $\xi^{(C)}, A$ and $B$, by assuming a functional dependence that takes in account the periodic boundary conditions). In all cases we have computed the statistical errors by using a standard jack-knife procedure. We have also computed $\lambda$ dependent correlation length estimators. By averaging them in the plateau region we have obtained another estimate of $\xi^{(C)}$, which turns out to be completely compatible with the one coming from the global fits. The fits turn out to be of very good quality, confirming the approximate validity of the form (20).

The correlation length of fig. 3 has quite a broad maximum close to $\beta=$ 1.35. $\xi^{(C)}$ close to its peak increases when going from $L=12$ to $L=24$, but for larger lattices it remains constant.

In fig. 4 we plot the maximum value of the correlation length, $\xi_{m}^{(C)}$, as a function of $\frac{1}{L}$, to stress the saturation that occurs for large $L$. The asymptotic $\xi_{m}^{(C)}$ is of order 4.5. It is rather consistent that the correlation length becomes independent from the size for sizes 3 to 4 times larger than the correlation length. 
In fig. 5 we plot the coefficient $B$ (i.e., the constant asymptotic value of the correlation function) computed from the fit to $C(\lambda)$ as a function of $\beta$. For large volumes $B$ should become identical with $m^{2}$ (which, in our analysis, turns out to be very similar to $m_{M}^{2}$ ), but its finite size corrections are smaller, especially in the high temperature region, where $B$ and $m_{M}^{2}$ are asymptotically zero. $B$ seems to take a non-zero expectation value starting from $\beta_{2} \simeq 1.35$. This method gives a very good estimate of the value of the critical temperature where $m_{M}^{2}$ becomes sizably different from zero.

In the region where $m_{M}^{2}$ is zero all different solutions of the mean field equations should become locally equal in the infinite volume limit, or more precisely their absolute difference should be in average go to zero with the volume.

It is natural to ask if these values of $\beta$ do correspond in the thermodynamical limit to real phase transitions. The quantity $W^{2}$ becomes different from zero as soon as there exist a realization of the magnetic field that admits two solutions. An explicit computation shows that if $h(i)=(-1)^{x+y+z}$ one finds two solutions when $\beta \geq \beta_{G} \simeq 1.015^{1}$. Simple minded arguments (which generalize the original Griffiths theorem for random diluted magnetic systems) suggest that the free energy is $C^{\infty}$ but not analytic at $\beta_{G}$

For $\beta>\beta_{G}$ there exist realizations of the magnetic field for which at least two solutions exist. These field configurations are special, and their measure is small. We expect therefore that $W^{2}$ is different from 1 , mathematically speaking, for $\beta \geq \beta_{G}$, but it becomes sizably different from 1 only at $\beta \geq \beta_{1}$. Similar arguments can be done for $\beta_{2}$. The non vanishing of $m_{M}^{2}$ for $\beta \geq \beta_{G}$ is a pathology that arises from our choice of considering only the maximal solution. If we consider the physically relevant quantity, i.e.,

$$
m^{2}=\sum_{\alpha} w_{\alpha}\left(m^{\alpha}\right)^{2}
$$

it should become different from zero only at values of $\beta$ much higher than $\beta_{G}$. The fact that the correlation length remains finite and somewhat small near $\beta_{2}$ may be taken as an indication that the true ferromagnetic transition at which $m^{2}$ becomes different from zero is at higher values of $\beta$.

The situation would be clarified if we could compute the full expression for $C(x)$, summing over all the solutions, but we have left this task for a

\footnotetext{
${ }^{1}$ An approximate formula valid for small $\mathcal{H}$ is $\beta_{G}=1+\frac{\mathcal{H}^{2}}{144}+O\left(\mathcal{H}^{4}\right)$.
} 
future work.

\section{Numerical Results for the Response Func- tions}

To compute the correlation functions in the mean field approach we must use the fluctuation-dissipation theorem. We are therefore lead to consider the susceptibility function $\chi_{i, j}$, which is equal to the derivative of the magnetization $m_{i}$ with respect to the field $h_{j}$ (for sake of typographical clearness in the following we will omit the solution label $\alpha$ ). If there is a single stable state we have to perturb the unique solution of equation (9). We get in this way the equation

$$
\chi_{i, j}=\beta\left(1-m_{i}^{2}\right)\left(\mathcal{D} \chi_{i, j}+\delta_{i, j}\right) .
$$

This is a linear sparse equation that can be solved by using standard iterative techniques.

The computation of $\chi$ for all the value of $i$ and $j$ would be extremely time consuming, so we compute the Green functions $g_{i} \equiv \chi_{i, 0}$ by setting $j=0$ and iterating the relation

$$
g_{i}=\beta\left(1-m_{i}^{2}\right)\left(\mathcal{D} g_{i}+\delta_{i, 0}\right) .
$$

We also compute the susceptibility $\chi \equiv \frac{1}{V} \sum_{i, j} \chi_{i, j}$ by iterating

$$
\begin{aligned}
\chi_{i} & =\beta\left(1-m_{i}^{2}\right)\left(\mathcal{D} \chi_{i}+1\right) \\
\chi & \equiv \frac{1}{V} \sum_{i=1}^{V} \chi_{i}
\end{aligned}
$$

and the overlap susceptibility $\chi^{q} \equiv \frac{1}{V} \sum_{i, j} \chi_{i, j} m_{i} m_{j}$ from

$$
\begin{aligned}
\chi_{i}^{q} & =\beta\left(1-m_{i}^{2}\right)\left(\mathcal{D} \chi_{i}^{q}+m_{i}\right) \\
\chi^{q} & \equiv \frac{1}{V} \sum_{i=1}^{V} \chi_{i}^{q} m_{i} .
\end{aligned}
$$


The name overlap susceptibility arises from the following considerations. Let us consider two replicas ( $\sigma$ and $\tau$ ) of the same system whose dynamics is determined by a Hamiltonian that contains the usual one system contribution plus a direct coupling among the two systems. The total Hamiltonian is

$$
H[\sigma]+H[\tau]-\epsilon \sum_{i} \sigma_{i} \tau_{i}
$$

This construction is common in the study of other disordered systems like spin glasses. The quantity $\chi^{q}$ coincide with $\frac{\partial q}{\partial \epsilon}$, evaluated at $\epsilon=0$, where $q$ is the overlap density, i.e., $\frac{1}{V} \sum_{i} \sigma_{i} \tau_{i}$.

In the interesting case in which the mean field equations admit many solutions $\alpha$ we follow the simplest procedure of weighting each of these with the weight $w^{\alpha}$ (we remind that we are taking in account only the two maximal solutions). In this way we are obtaining only one term of the two that form the full susceptibility. It is easy to check that the response function

$$
R(i, j) \equiv \frac{\partial}{\partial h_{j}} \sum_{\alpha} w_{\alpha} m_{i}^{\alpha}
$$

is given by

$$
R(i, j)=\chi_{i, j}+\beta\left(\sum_{\alpha} w_{\alpha} m_{i}^{\alpha} m_{j}^{\alpha}-\sum_{\alpha} w_{\alpha} m_{i}^{\alpha} \sum_{\gamma} w_{\gamma} m_{j}^{\gamma}\right) .
$$

The second term (in brackets), which arises in presence of many solutions, is likely to be dominant near the critical point, as will shall see below. It may be convenient to call the first the diagonal contribution, and the second one the off-diagonal contribution.

We have computed the diagonal contributions $\chi$ and $\chi^{q}$ with the results shown in figs. 6 and 7. It is impressive that $\chi$ has a sharp maximum close to $\beta_{1}$, while $\chi^{q}$ has a peak at much higher beta (slightly above $\beta_{2}$ ) and does not show any significant anomaly at $\beta_{1}$. These two peaks are volume independent for large volume. The correctness of this result is confirmed by the direct analysis of the correlation length corresponding to $\chi, \xi_{\chi}^{(1)}$, which we show in fig. 8. $\xi_{\chi}^{(1)}$ does never become large in the whole region and for $\beta \leq \beta_{1}$ essentially coincides with $\xi^{(C)}$, the correlation length we have discussed in the previous section. We find that the supersymmetry prediction of equality of the two correlation lengths is correct in the region $\beta \leq \beta_{1}$ where only one solution is present. 
We have also considered the correlation lengths $\xi_{\chi}^{(n)}$ defined by taking the $n$-th power of the zero bi-momentum correlation functions that give $\xi_{\chi}^{(1)}$, and by looking at their decay. They do not present a significant difference (once divided by $n$ ) from the one obtained for $n=1$. In fig. 9 we show the correlation length with $n=2$ (which has the smallest statistical error), which can be compared with the $n=1$ result of fig. 8 . The two sets of curves are very similar.

Evaluating at least some approximation to the off-diagonal contribution to the susceptibility is essential. We have done it by only using our maximal solutions. There is a large statistical error. In the low temperature region we expect that the off-diagonal contribution is proportional to $N^{1 / 2}$, this contribution arising from a few exceptional configurations of the magnetic field that have two solutions with opposite magnetization with similar weight. This event happens with a probability of order $1 / N^{1 / 2}$; the corresponding off-diagonal susceptibility is of order $N$, so that the net contribution to the susceptibility coming from these exceptional configurations is proportional to $N^{1 / 2}$. In this region the off-diagonal susceptibility is dramatically increasing, showing the trend to diverge about $\beta \simeq 1.30$. Anyhow there is no convincing argument that implies that the restriction to the maximal solution should be a good approximation, apart from very close to $\beta_{1}$, where only two stable solutions are expected.

A full computation (including all the solutions) of both the diagonal and the non-diagonal contribution to the susceptibility would be extremely interesting.

\section{Conclusions}

The existence of many solutions to the mean field equations turns out to be a crucial phenomenon; inside a single solution (at least of the maximal type) one does not see any sign of the presence of a divergent correlation length. The critical behavior of the susceptibility and of the correlation length in a $3 d$ RFIM is dominated by the effects of the presence of many solutions. The supersymmetric predictions start to fail exactly at the point where one finds more than one solution of the mean field equations. It is not surprising that dimensional reduction, which completely misses the existence of more than one solution, gives unreliable exponents at the critical point. 
It is reasonable that each solution of the mean field equation does correspond to a valley for the energy in configuration space ${ }^{2}$. In this case the dynamics of Monte Carlo simulations of a real system also at temperature slightly above the critical one is likely to be dominated by thermal activated tunnelling among different valleys, and we expect it to be a slow process.

\section{Acknowledgements}

We thank M. Aizemann, M. Mezard and N. Sourlas for many interesting discussions.

\footnotetext{
${ }^{2}$ We reserve the word state for solutions (or cluster of solutions) such that their distance $\frac{1}{V} \sum_{i}\left(m_{i}^{\alpha}-m_{i}^{\gamma}\right)^{2}$ does not vanish in the infinite volume limit.
} 


\section{References}

[1] D. P. Belanger and A. P. Young, The Random Field Ising Model, Santa Cruz University preprint, 1991.

[2] Y. Shapir, The Random Field Enigma, Rochester University preprint, 1991, to appear in Recent Progress in Random Magnets.

[3] G. Parisi, in Recent Advances in Field Theory and Statistical Mechanics, edited by J.-B. Zuber and R. Stora (North-Holland, Amsterdam, Netherlands, 1984), reprinted in G. Parisi, Field Theory, Disorder and Simulations (World Scientific, Singapore 1992); G. Parisi, in Quantum Field Theory and Quantum Statistics. Essays in Honour of the Sixtieth Birthday of E.S. Fradkin, edited by I. A. Batalin, C. J. Isham and G. A. Vilkovisky, (Adam Hilger, Bristol, U.K., 1987).

[4] A. P. Young, J. Phys. C 100 (1977) L257.

[5] J. Z. Imbrie, Phys. Rev. Lett. 53 (1984) 1747.

[6] G. Parisi and N. Sourlas, Phys. Rev. Lett. 43 (1979) 744;

[7] A. T. Ogielski, Phys. Rev. Lett. 57 (1986) 1251.

[8] V. Dotshenko and G. Parisi, J. Phys A, to be published.

[9] M. Mezard and P. Young, Europhys. Lett. 18 (1992) 653.

[10] H. Yoshizawa and D. P. Belanger, Phys. Rev. B30 (1984) 5220; C. Ro, G. S. Grest, C. M. Soukoulis and K. Levin, Phys. Rev. B31 (1985) 1682; C. S. Grest, C. M. Soukoulis and K. Levin, Phys. Rev. B 33 (1986) 7659.

[11] A. P. Young and M. Nauenberg, Phys. Rev. Lett. 54 (1985) 2429.

[12] M. Aizemann, private communication. 


\section{Figure Captions}

1. $W^{2}$ as a function of $\beta$. dots for the $12^{3}$ lattice, dashes-dots for the $24^{3}$ lattice, dashes for the $36^{3}$ lattice and solid line for the $48^{3}$ lattice.

2. As in fig. 1 , but $m_{M}^{2}$.

3. The inverse correlation length $m \equiv \frac{1}{\xi^{(C)}}$ as a function of $\beta$ for different lattice sizes.

4. The maximum correlation length $\xi_{m}^{(C)}$ as a function of the inverse linear size of the system.

5. The constant coefficient $B$ from the global fit to $C(\lambda)$ as a function of $\beta$.

6. As in fig. 1, but $\chi$, the diagonal contribution to the susceptibility.

7. As in fig. 1, but $\chi^{q}$, the diagonal contribution to the overlap susceptibility.

8. As in fig. 1 , but $\xi_{\chi}^{(1)}$.

9. As in fig. 1 , but $\xi_{\chi}^{(2)}$. 


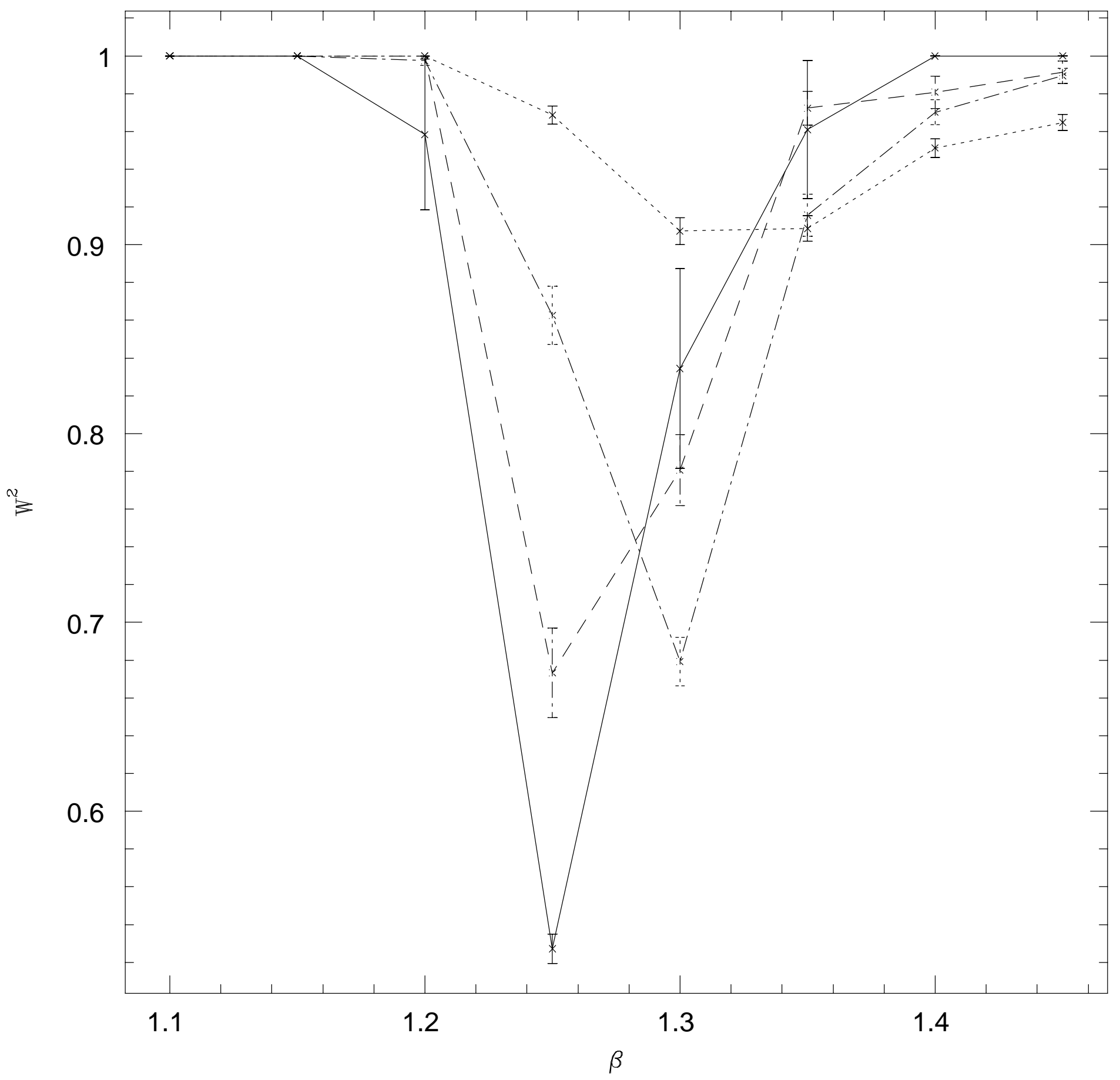




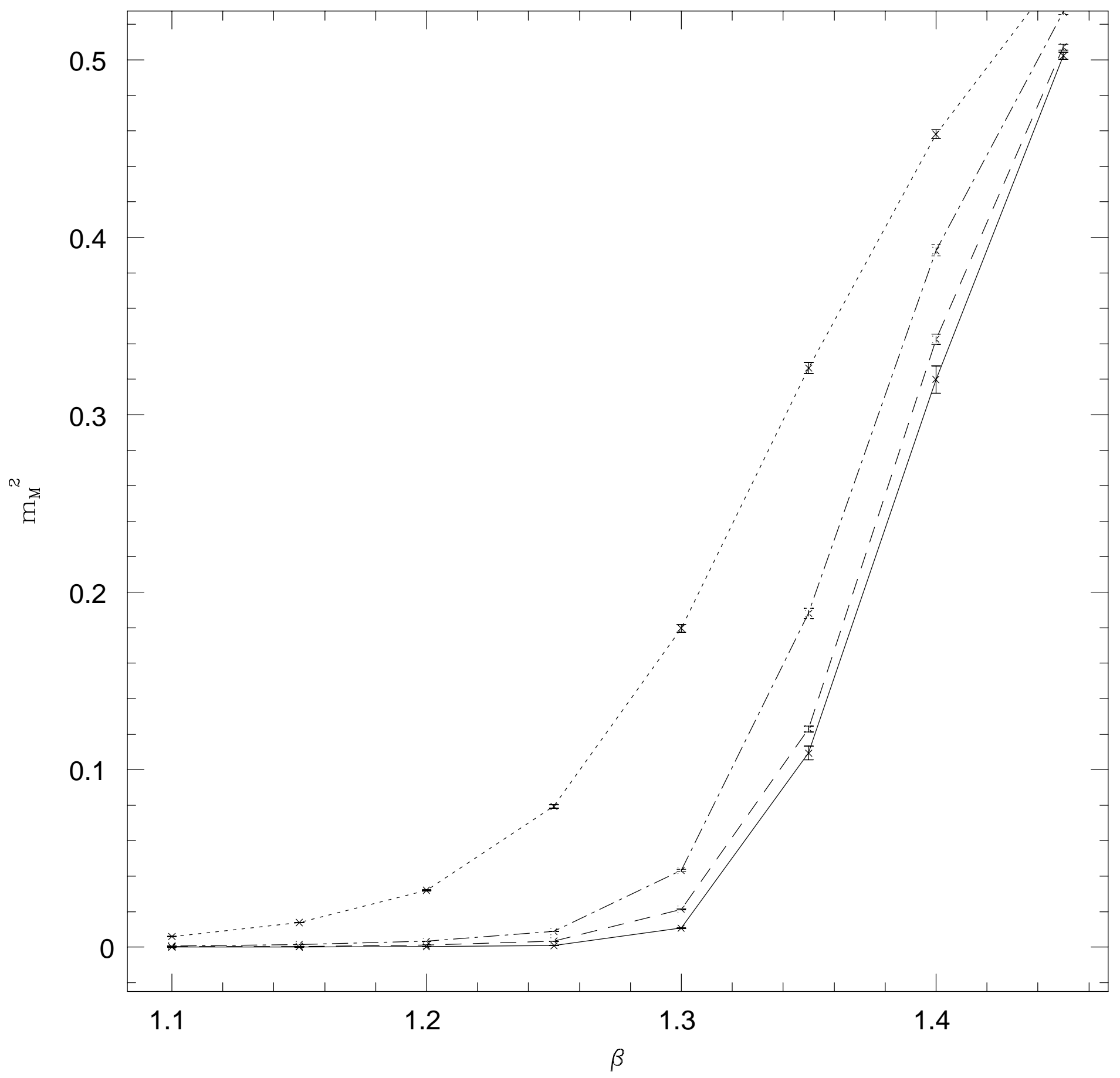




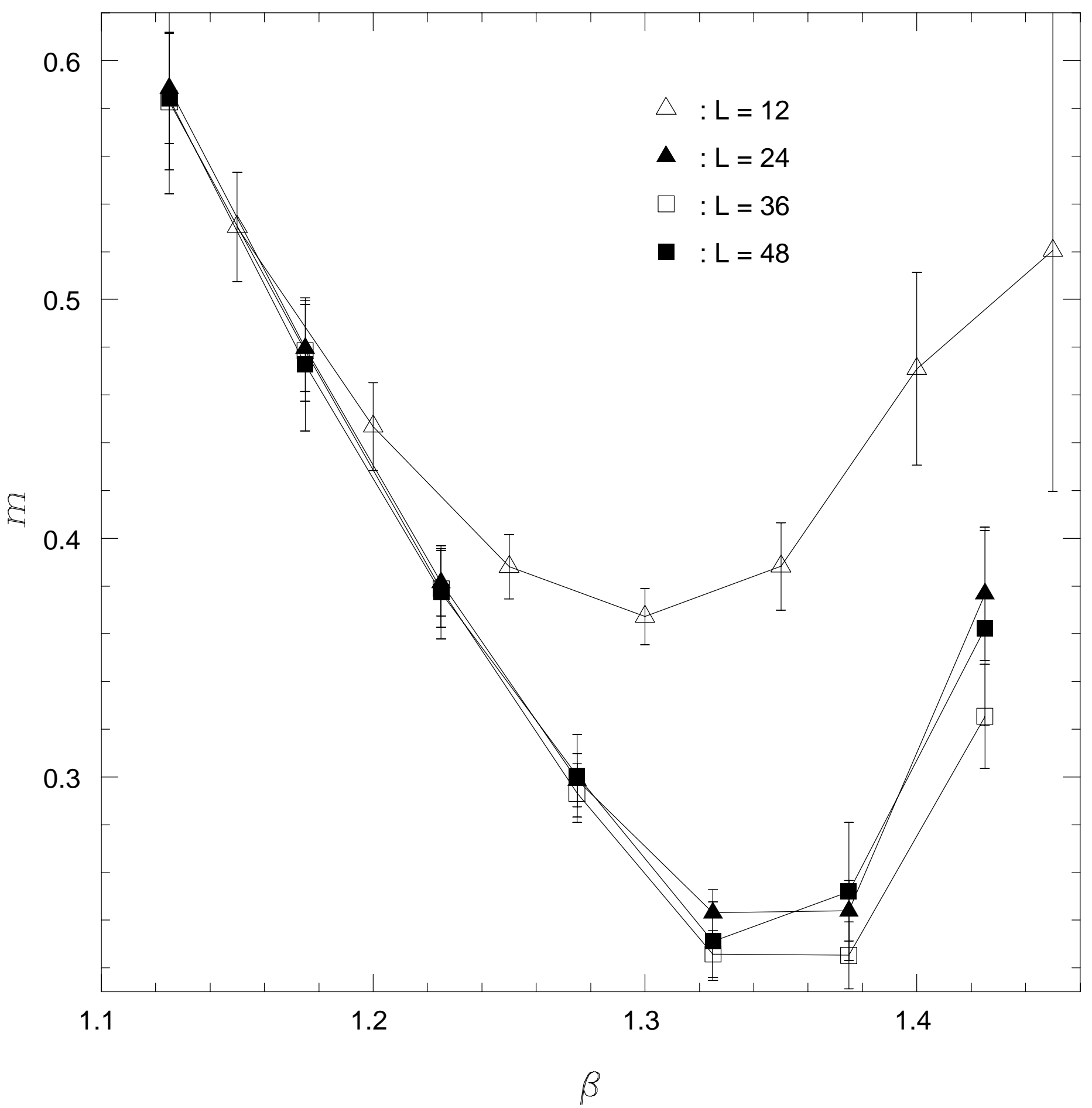




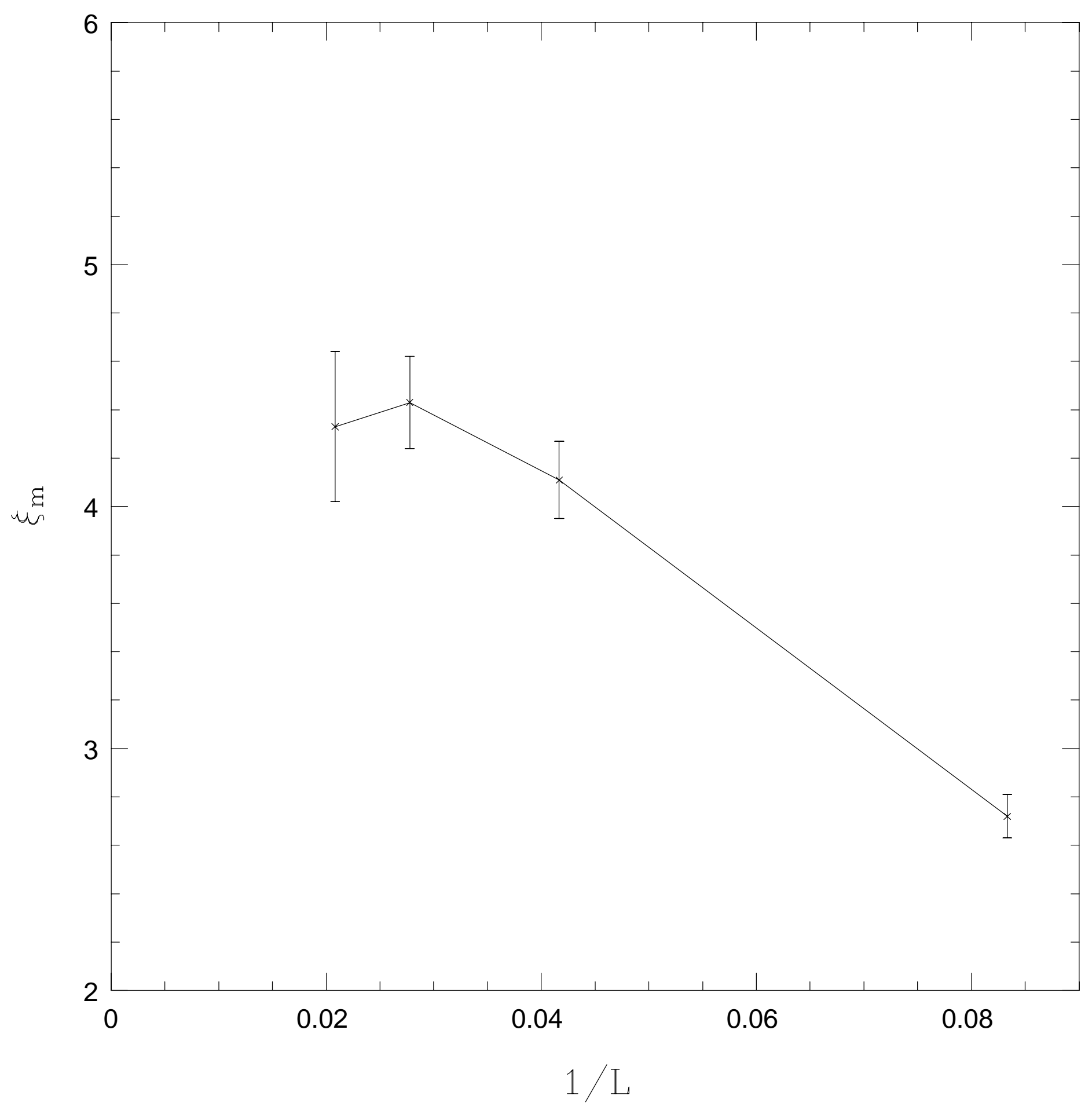




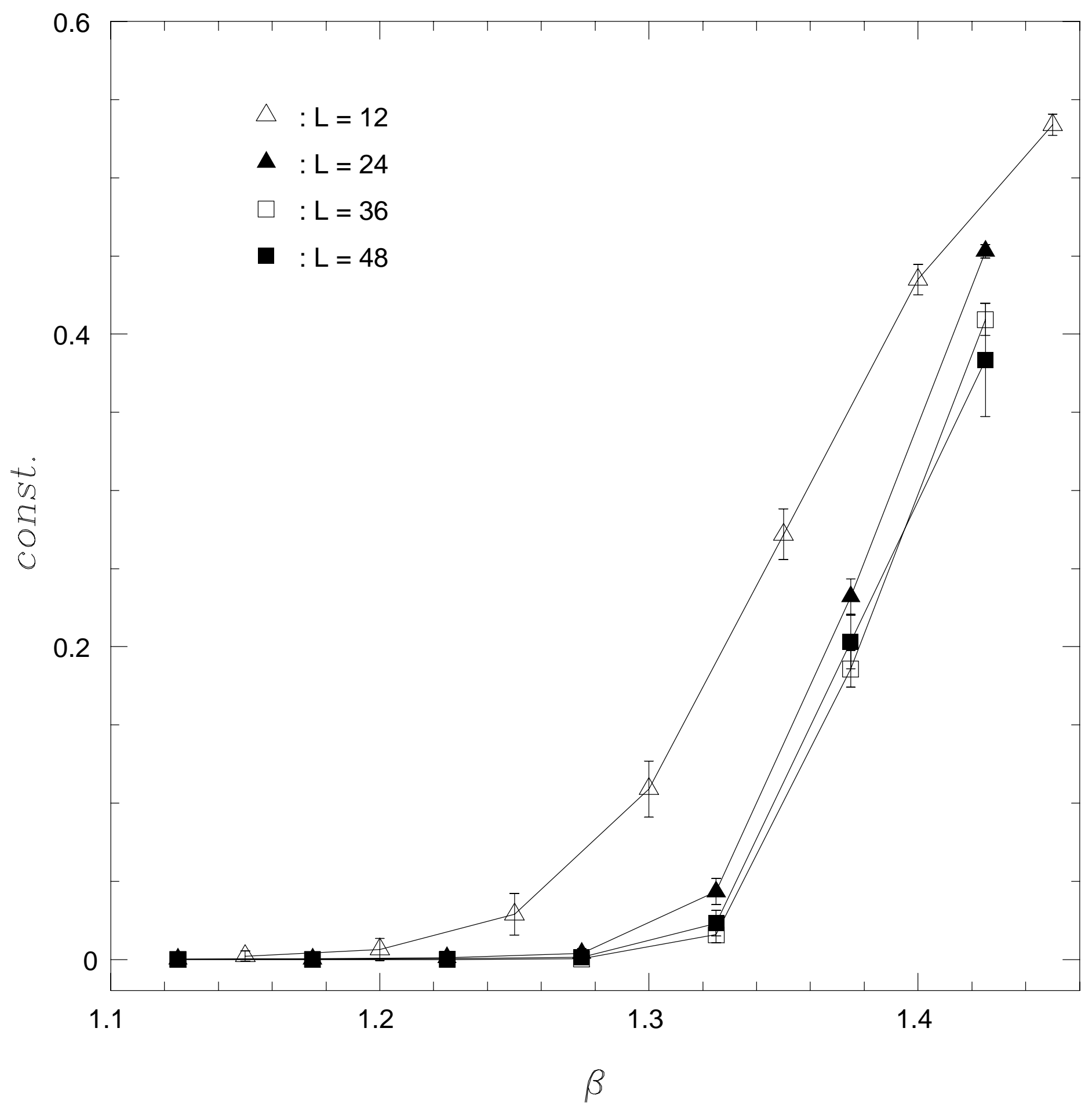




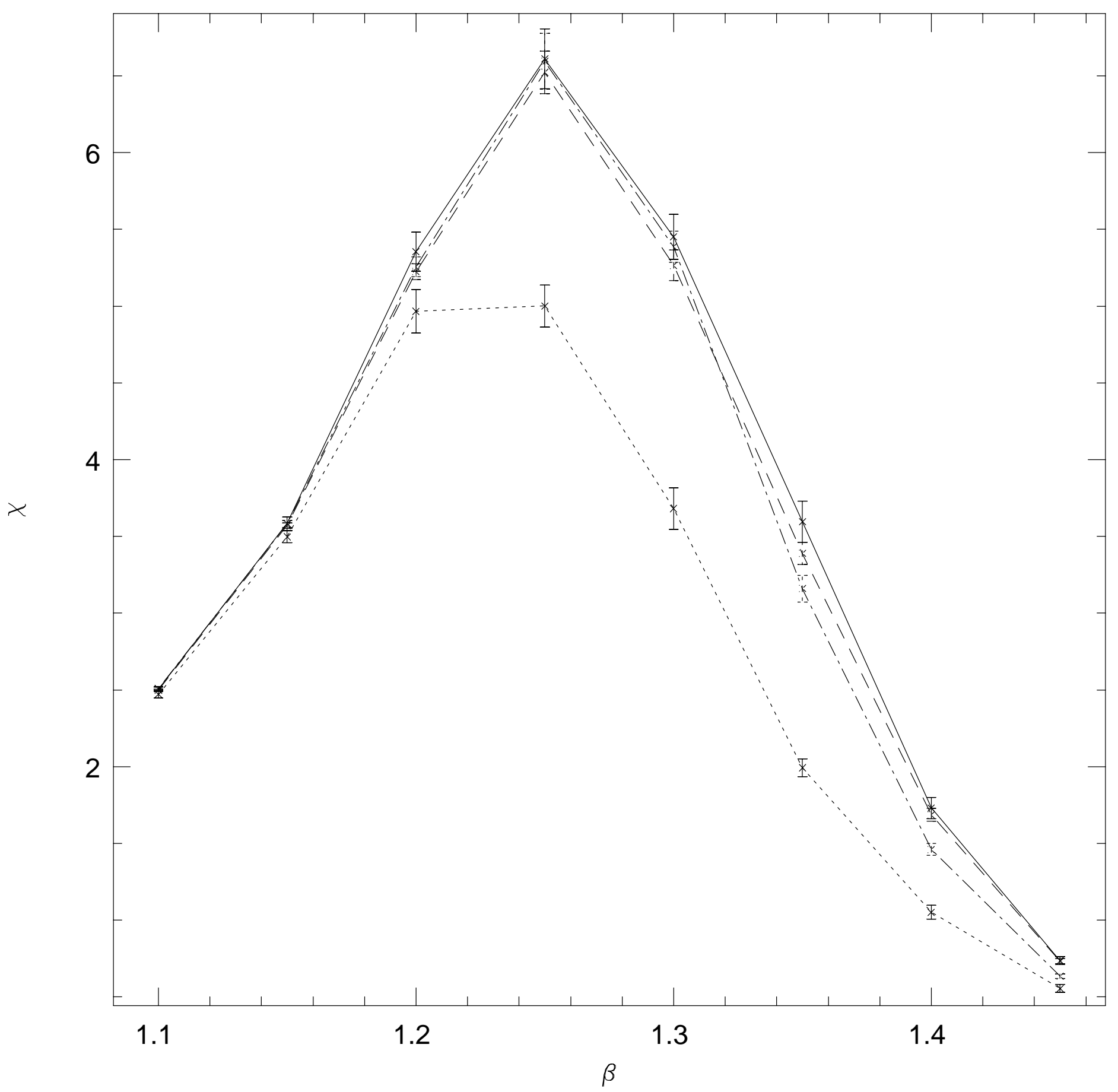




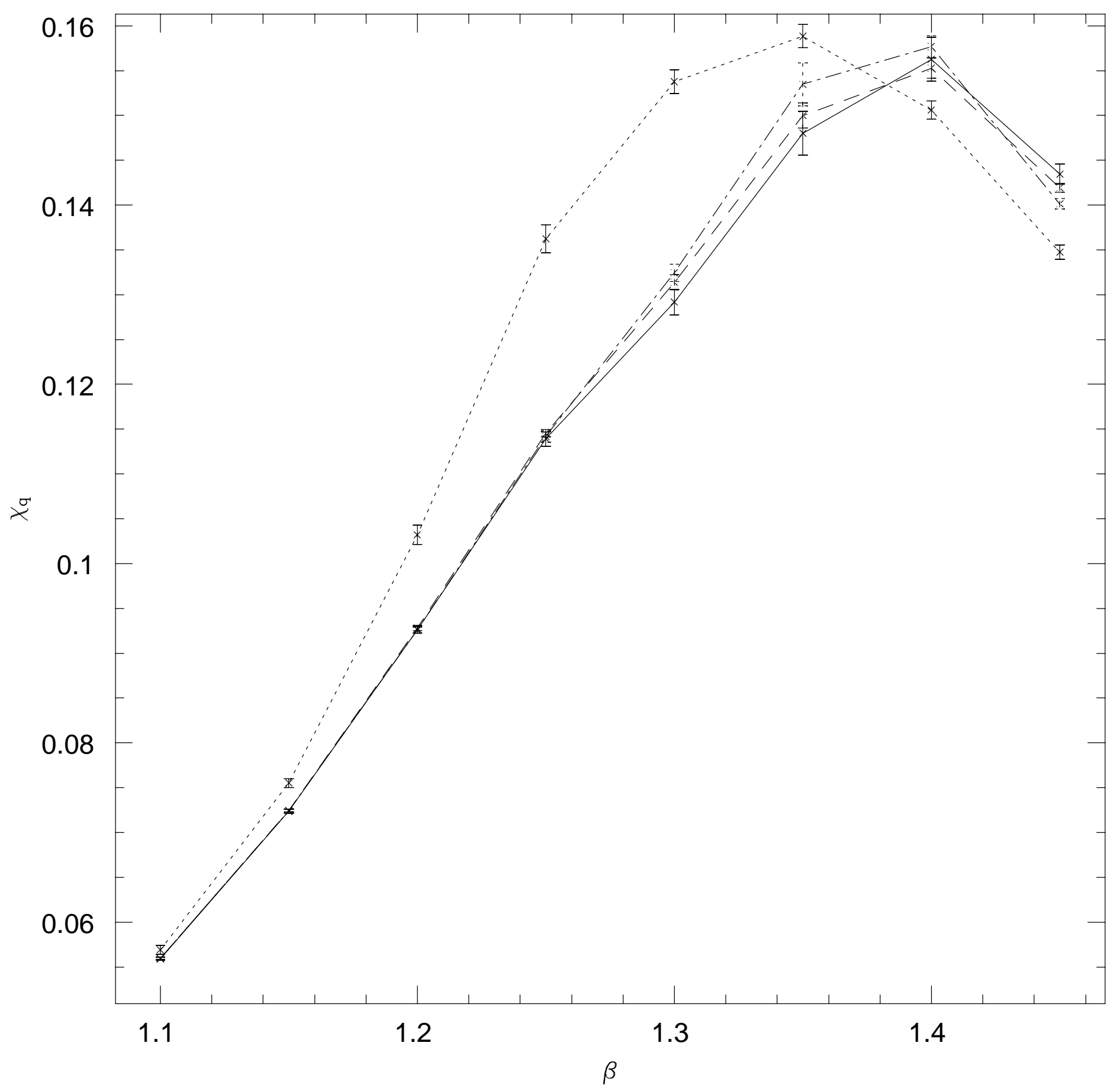




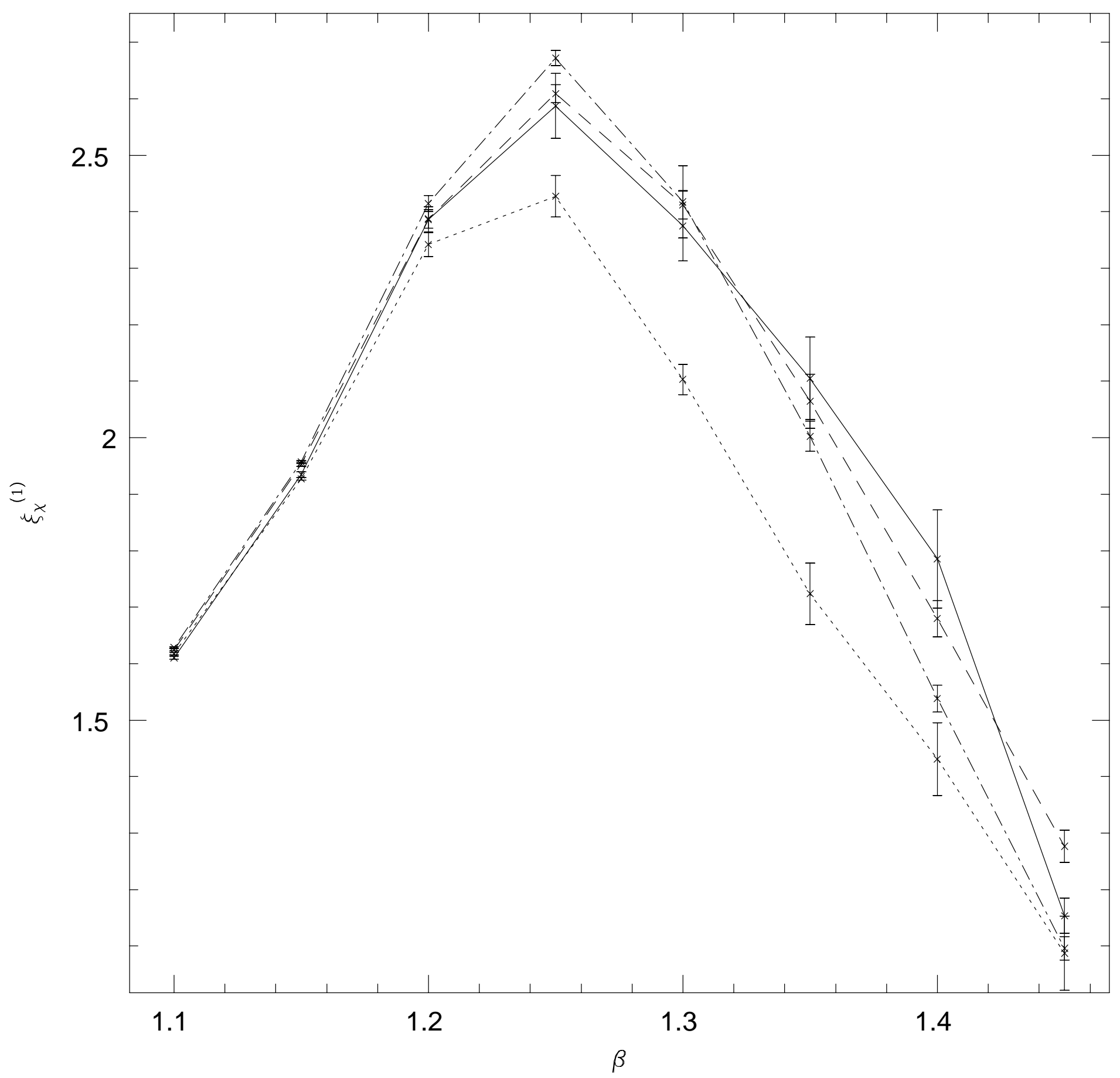




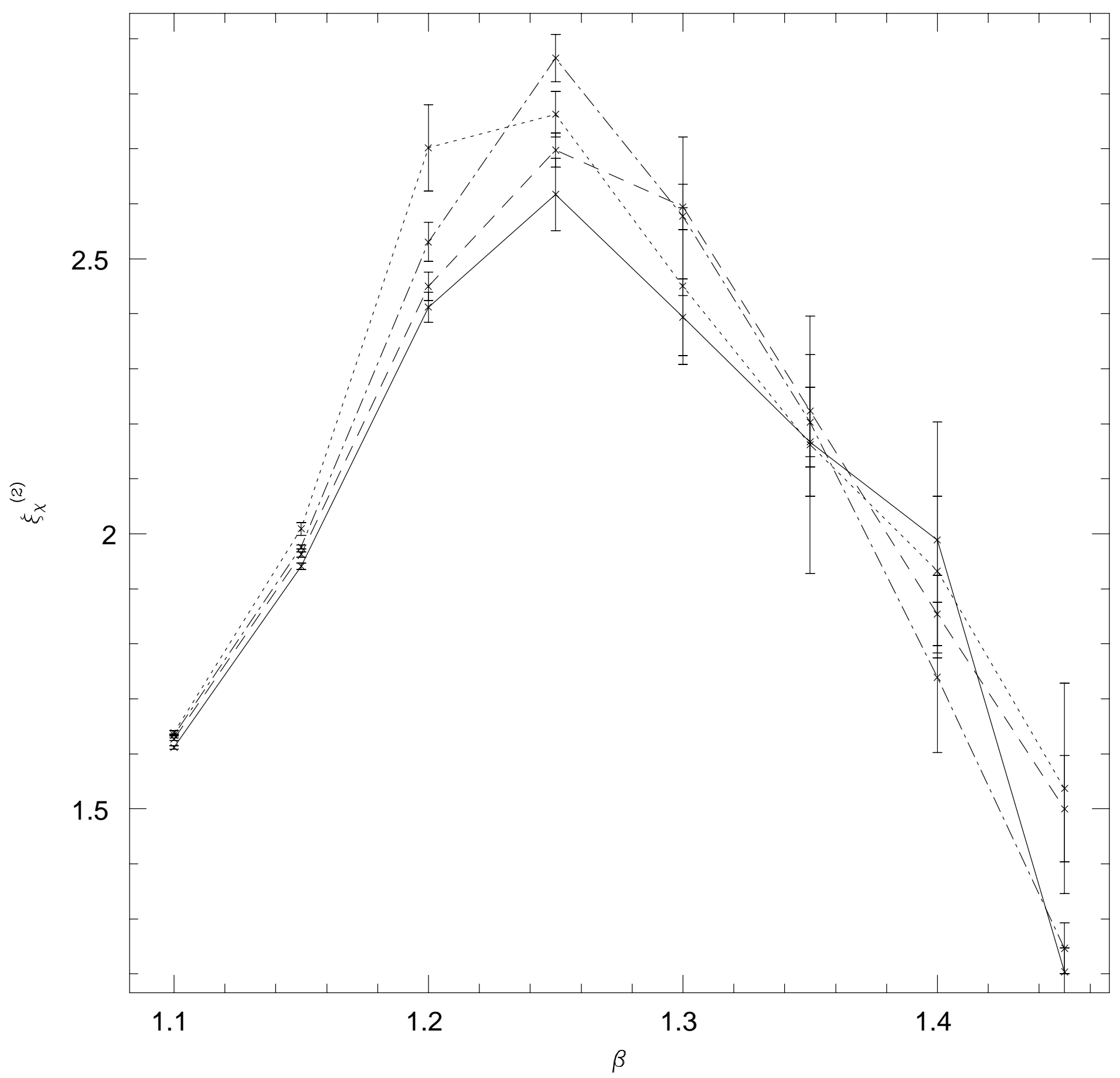

